\title{
Epithelial-to-mesenchymal transition (EMT) induced by inflammatory priming elicits mesenchymal stromal cell-like immune- modulatory properties in cancer cells
}

\author{
M Ricciardi ${ }^{1}$, M Zanotto ${ }^{1}$, G Malpeli ${ }^{2}, \mathrm{G}$ Bassi ${ }^{1}$, O Perbellini ${ }^{1}, \mathrm{M}$ Chilosi $^{3}$, F Bifari ${ }^{1}$ and M Krampera ${ }^{*}, 1$ \\ ${ }^{1}$ Stem Cell Research Laboratory, Section of Hematology, Department of Medicine, University of Verona, Verona 37134, Italy; \\ ${ }^{2}$ Department of Surgery, University of Verona, Verona 37134, Italy and ${ }^{3}$ Department of Pathology and Diagnostics, Section of \\ Pathological Anatomy, University of Verona, Verona 37134, Italy
}

Background: Epithelial-to-mesenchymal transition (EMT) has a central role in cancer progression and metastatic dissemination and may be induced by local inflammation. We asked whether the inflammation-induced acquisition of mesenchymal phenotype by neoplastic epithelial cells is associated with the onset of mesenchymal stromal cell-like immune-regulatory properties that may enhance tumour immune escape.

Methods: Cell lines of lung adenocarcinoma (A549), breast cancer (MCF7) and hepatocellular carcinoma (HepG2) were co-cultured with T, B and NK cells before and after EMT induction by either the supernatant of mixed-lymphocyte reactions or inflammatory cytokines.

Results: EMT occurrence following inflammatory priming elicited multiple immune-regulatory effects in cancer cells resulting in NK and T-cell apoptosis, inhibition of lymphocyte proliferation and stimulation of regulatory $T$ and $B$ cells. Indoleamine 2,3dioxygenase, but not Fas ligand pathway, was involved at least in part in these effects, as shown by the use of specific inhibitors.

Conclusions: EMT induced by inflammatory stimuli confers to cancer cells some mesenchymal stromal cell-like immunemodulatory properties, which could be a cue for cancer progression and metastatic dissemination by favouring immune escape.

Epithelial-to-mesenchymal transition (EMT) is a well-described pathogenetic event in cancer progression and metastasis, as cancer cells undergoing EMT acquire highly invasive features supporting cancer spreading. Different inducers of EMT have been identified, including transforming growth factor-beta (TGF- $\beta$ ), tumour necrosis factor-alpha (TNF- $\alpha$ ) and Notch, Hedgehog and Wnt molecular pathways. All these stimuli induce EMT by upregulating trascription factors, such as Snail, Twist and Zeb1, which promote cell polarity loss by destroying tight junctions and degrading adhesion molecules, such as E-cadherin (Mantovani et al, 2008; Klymkowsky and Savagner, 2009; Thiery et al, 2009; Voulgari and Pintzas, 2009; Vetter et al, 2010; Kotsafti et al, 2012; Liu et al, 2013).
EMT process is tightly connected to inflammation. In fact, inflammatory responses have critical roles in all the stages of tumour development, such as cancer initiation, promotion, malignant phenotype acquisition, intravascular dissemination and metastasis (Mantovani et al, 2008). In addition, inflammation has been recently identified as a key inducer of EMT during cancer progression; for this reason, EMT is now accounted for linking inflammation and cancer through microenvironmental modifications capable of driving tumour progression (Mantovani et al, 2008; López-Novoa and Nieto, 2009; Grivennikov et al, 2010).

The link between EMT and cancer immune escape is still only partially uncovered, including the mechanisms through which 
cancer cells may overcome specific immune responses or even take advantage from the immune reaction for their own survival and growth. However, there is a number of evidence in favour of this link. For instance, the upregulation of some EMT-inducing factors, such as Snail1, may induce the expression of pro-inflammatory interleukins (IL-1, IL-6 and IL-8) (Ge et al, 1999), but at the same time it may trigger some immunosuppressive mechanisms based on inhibitory cytokines, regulatory $\mathrm{T}$ cells, impaired dendritic cell functions and resistance to cytotoxic T cells, thus accelerating the onset of cancer metastasis (Kudo-Saito et al, 2009; Grivennikov et al, 2010). In addition, some cancer cell lines may downregulate in a TGF- $\beta$-dependent manner a number of effector molecules in $\mathrm{CD}^{+} \mathrm{T}$ cells, such as granzyme B, perforin and Fas ligand (FasL), thus suppressing the generation of cytotoxic effector cells and favouring the expansion of regulatory CD4 ${ }^{+} \mathrm{T}$ cells (Joffroy et al, 2010). Finally, cancer cells may upregulate indoleamine 2,3dioxygenase (IDO) enzyme and therefore drive effector lymphocytes (mainly $\mathrm{T}$ cells) towards either cell cycle arrest or apoptosis (Uyttenhove et al, 2003; Muller et al, 2005; Lob et al, 2009a).

To better clarify the relationship between EMT and immune escape, we investigated how neoplastic cells interact with the immune effector cells (T, B and NK cells) before and after EMT induction in epithelial cancer cell lines through inflammatory priming. Our work hypothesis was that the inflammation-induced acquisition of mesenchymal phenotype by neoplastic epithelial cells could be associated with the onset of immune-regulatory properties commonly displayed by mesenchymal stromal cells (MSCs) following inflammatory priming, including IDO activation (Krampera, 2011), thus enhancing the possibility of tumour immune escape. In other words, MSCs and cancer epithelial cells undergoing EMT in a inflammatory microenvironment could share a common pattern of regulatory mechanisms favouring their survival.

\section{MATERIALS AND METHODS}

Cell culture and EMT induction. A549 cells (lung adenocarcinoma), MCF-7 cells (breast cancer) and HepG2 (hepatocarcinoma) cells were grown at $37^{\circ} \mathrm{C}$ in $5 \% \mathrm{CO}_{2}$ atmosphere in DMEM (Sigma-Aldrich, Steinheim, Germany) supplemented with $1 \%$ Penicillin/streptomicyn (Invitrogen, Pasley, UK) and 10\% fetal calf serum (Invitrogen); this culture condition represented the control (CTRL).

EMT was induced by the supernatant derived from a one-way, $1: 1 \mathrm{E} / \mathrm{T}$ ratio mixed lymphocyte reaction (MLR) in fresh culture medium or by adding the combination of TGF- $\beta\left(10 \mathrm{ng} \mathrm{ml}^{-1}\right)$, interferon-gamma (IFN- $\gamma)\left(10 \mathrm{ng} \mathrm{ml}^{-1}\right)$ and TNF- $\alpha\left(10 \mathrm{ng} \mathrm{ml}^{-1}\right)$ to the culture medium (MIX). In both cases, cell stimulation lasted $48 \mathrm{~h}$.

RNA isolation and quantitative PCR. RNA was extracted with TRIzol (Invitrogen) and used for quantitative PCR (qPCR) according to the established procedures. The primers used were:

E-cadherin, forward: 5'-GACACCAACGATAATCCTCCGA-3', reverse: 5'-GGCACCTGACCCTTGTACGT-3';

Vimentin, forward: 5'-TCCAAGTTTGCTGACCTCTCTG-3', reverse: 5'-CAGTGGACTCCTGCTTTGCC-3';

Snaill, forward: $5^{\prime}$-CCCAGTGCCTCGACCACTAT- ${ }^{\prime}$, reverse: 5'-GCTGGAAGGTAAACTCTGGATTAGA-3';

Snail2, forward: 5' -TGCATATTCGGACCCACACA-3', reverse: 5'-TGTTGCAGTGAGGGCAAGAA-3';

Zeb1, forward: 5'-GATCCAGCCAAATGGAAATCA-3', reverse: 5'-GGCGGTGTAGAATCAGAGTCATTC-3';

Ido1, forward: $5^{\prime}$-GCTAAAGGCGCTGTTGGAAA-3' ${ }^{\prime}$, reverse: 5'-GGGTTCACATGATCGTGGATT-3'; and $\beta$-actin, forward: $5^{\prime}$-CGCGAGAAGATGACCCAGAT-3', reverse: 5'-GTCACCGGAGTCCATCACG-3' .

Cancer cell characterisation. The following antibodies were used to characterise cancer cell immunophenotype before and after EMT induction: anti-CD324-PE (Biolegend, San Diego, CA, USA), anti-vimentin (R\&D System, Minneapolis, MN, USA), anti-HLA-A,B,C-PE (BD Bioscience, San Jose, CA, USA), antiCD54-PE (BD Bioscience), anti-HLA-DR-PE (BD Bioscience), and corresponding isotype controls. Cells were stained according to the manufacturer's instructions and analysed by FACSCanto II (BD Bioscience). For immunofluorescence, cancer cells were immunostained with the following antibodies: E-cadherin (Abcam, Cambridge, MA, USA), Vimentin (Dako, Glostrup, Denmark), relative secondary antibody (Jackson Immunoresearch, West Grove, PA, USA), and Topro3 (Invitrogen) for nucleus counterstaining. Images were acquired with the Zeiss LSM 510 (Zeiss, Oberkochen, Germany) confocal microscope.

Immune-regulatory properties of cancer cells. Human peripheral blood mononuclear cells were obtained from healthy blood donors after written informed consent, as approved by the Ethics Committee of Azienda Ospedaliera Universitaria Integrata Verona (N. Prog. 1828, 12 May 2010 - 'Institution of cell and tissue collection for biomedical research in Onco-Hematology'). CD3 ${ }^{+} \mathrm{T}$ cells, CD $19^{+} \mathrm{B}$ cells and $\mathrm{CD}^{+} 6^{+} \mathrm{NK}$ cells were purified from peripheral blood using appropriate negative selection kits (Miltenyi Biotec, Bergisch Gladbach, Germany) with at least 95\% cell purity, as evaluated by flow cytometry. Proliferation of stimulated B, T and NK cells in co-culture with cancer cells was evaluated at day +4 (B cells) and +7 ( $\mathrm{T}$ and NK cells) by means of CFDA-SE dilution method (Invitrogen), as previously described. Ratio between effector cells and cancer cells were 1:1 (NK or B:cancer cells) and 10:1 (T:cancer cells), according to previous setting experiments (Di Trapani et al, 2013).

All effectors were labeled with CFDA-SE ( $5 \mathrm{~mm}$ ) and then cocultured with irradiated cancer cell lines (7000 cGy). At the end of co-culture, only viable lymphocytes identified as $\mathrm{CD} 45^{+}$and Topro3 $^{-}$cells were analysed.

The mechanisms leading to proliferation arrest and apoptosis induction of immune effector cells were assessed by adding a specific IDO inhibitor (L-1-methyl-tryptophan (L-1MT), Sigma-Aldrich) (1 mM) to the co-cultures after T-cell stimulation and by evaluating FasL expression by flow cytometry and enzymelinked immunosorbent assay (ELISA).

Pro-apoptotic properties of cancer cells. The viability of T, B and NK cells co-cultured with irradiated cancer cell lines (7000 rad) was assessed by flow cytometry using the Annexin V/PI Kit (Miltenyi Biotec). Effector cells were recognised on the basis of their positivity for anti-human-CD45 and characterised as viable cells (annexin $\mathrm{V}^{-} / \mathrm{PI}^{-}$); dying cells (annexin $\mathrm{V}^{+} / \mathrm{PI}^{-}$) and dead cells (annexin $\mathrm{V}^{+} / \mathrm{PI}^{+}$).

$\mathrm{T}$ regulatory cell subsets. The presence of $\mathrm{T}$ regulatory cells subset was evaluated with the following antibodies: anti-CD45 PerCP (Miltenyi Biotec), anti-CD4APCH7 (BD Bioscence), antiCD8PECy7 (BD Bioscience), anti-CD25FITC (Miltenyi Biotec), anti-FoxP3PE (Miltenyi Biotec), and anti-ki67Alexa647 to track proliferation (eBioscience, San Diego, CA, USA).

$B$ regulatory cell subsets. The presence of $B$ regulatory cells subset was evaluated with the following antibodies: anti-ki67Alexa647 (eBioscience), anti-CD45PerCP (Miltenyi Biotec), anti-CD24FITC (Miltenyi Biotec), and anti-CD38APC (Miltenyi Biotec).

Statistical analysis. Data were expressed as mean \pm s.d. A one-way analysis of variance and Bonferroni's correction for multiple comparisons were used to determine statistical significance $(P<0.05)$. 


\section{RESULTS}

Induction of EMT in cancer cell lines through inflammatory priming. Cell lines of lung adenocarcinoma (A549), breast cancer (MCF7) and hepatocellular carcinoma (HepG2) were induced to undergo EMT by adding to the culture medium for $48 \mathrm{~h}$ either MLR supernatant or a cytokine combination (MIX) consisting of TGF- $\beta+$ IFN- $\gamma+$ TNF- $\alpha$, as described in Materials and methods section. Both stimuli resulted in capable of inducing EMT features in all the three cancer cell lines (Figure 1). The combination of the three cytokines as well as TGF- $\beta+$ TNF- $\alpha$ were mostly effective in inducing EMT. The effect of each cytokine alone or in combination with the others is described in Supplementary Figure S1. In particular, A549 cancer cells showed a significant transcription enhancement of snail 1 and snail2 genes and downmodulation of cdh1 gene (E-cadherin) expression following both MLR and MIX treatment. Vimentin transcript levels were significantly upregulated only with the MIX treatment (Figure 1A, left panel). At the protein level, flow cytometric analysis showed the significant reduction of E-cadherin expression and upregulation of ICAM-1 and HLA A, B, C proteins following MLR and MIX treatment. Again, vimentin protein was upregulated only with MIX treatment. No significant difference in HLA-DR protein expression was found (Figure 1A, middle panel). EMT-like morphological changes (from cubblestone to isolated cells), E-cadherin protein loss and vimentin upregulation were confirmed by in situ immunofluorescence (Figure 1A, right panel).
Similarly, MCF7 cancer cells treated with either MLR supernatant or cytokine MIX combination showed a significant increase of snail 1 and snail 2 transcripts, as compared with normal culture conditions. Vimentin and zeb1 gene expression was significantly upregulated only with MIX treatment while cdh1 gene expression was not changed (Figure 1B, left panel). At the protein level, both MLR and MIX treatment induced the significant reduction of E-cadherin expression, whereas ICAM-1, HLA-A, B, C and HLADR were upregulated (Figure 1B, middle panel). Again, EMT-like morphological changes, E-cadherin protein loss and vimentin upregulation were confirmed by in situ immunofluorescence (Figure 1B, right panel).

Finally, HepG2 cancer cells after MLR and MIX treatment showed a significant transcription enhancement of snail1, zeb1 and vimentin genes. Snail2 was upregulated only with MIX treatment, while $\mathrm{CDH} 1$ gene expression resulted downregulated only with MLR treatment, having MIX the opposite effect (Figure 1C, left panel). Flow cytometry showed a significant E-cadherin downmodulation and ICAM-1 expression increase after both MLR and MIX treatment. Vimentin protein upregulation was significantly higher with MIX treatment. The expression of HLA-A, B, C and HLA-DR was not changed by the treatments (Figure 1C, left panel). In situ immunofluorescence confirmed the EMT-like morphological changes, E-cadherin protein loss and the slight vimentin upregulation observed by flow cytometry (Figure 1B, right panel).

Cancer cell effects on NK cells following EMT. A549, MCF7 and HepG2 cells, either at basal conditions or after EMT induction with
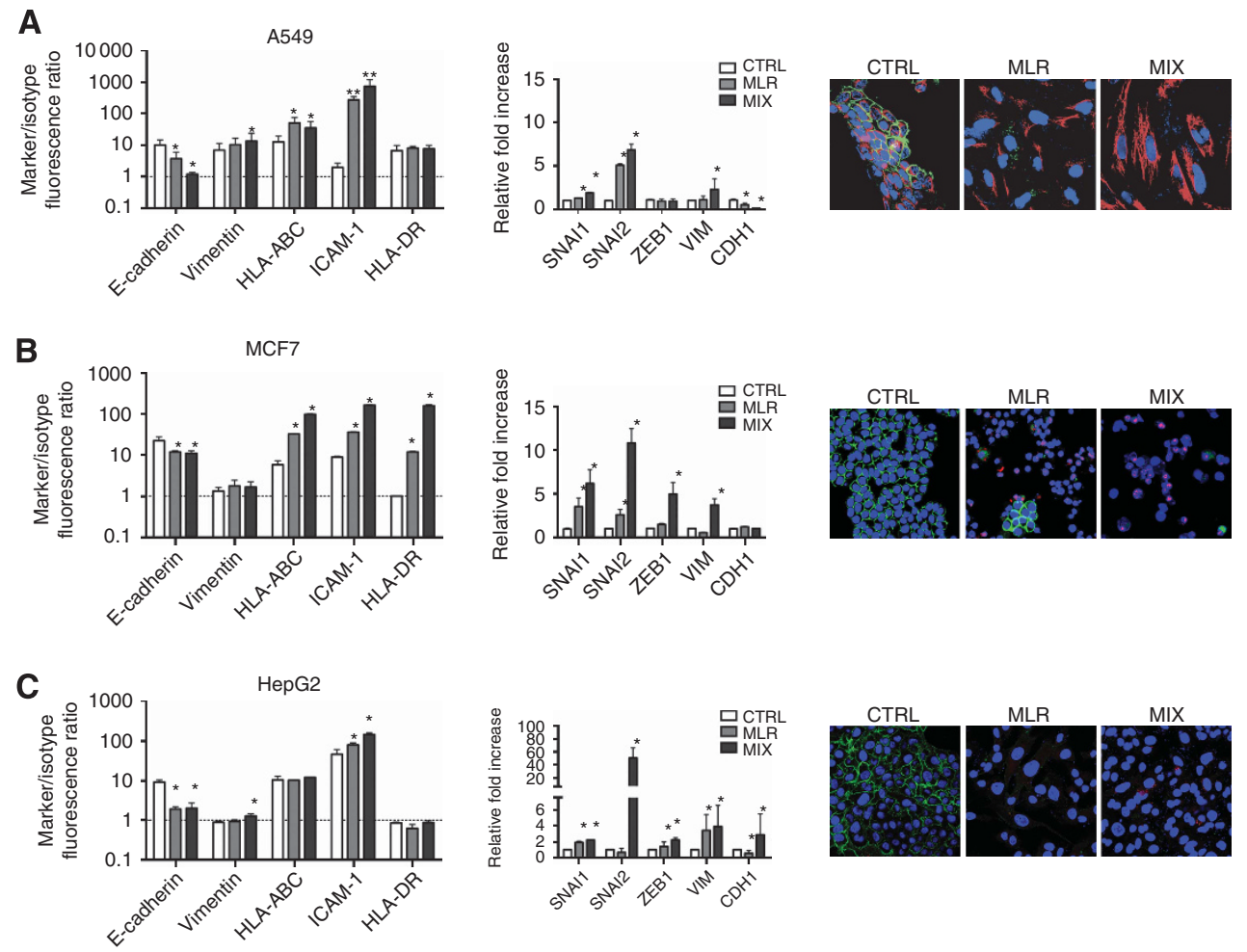

Figure 1. Evaluation of EMT changes in three cancer cell lines. Left panel: Evaluation of EMT changes by qRT-PCR on (A) A549, (B) MCF7 and (C) HepG2 cancer cell lines in control medium (CTRL, white columns) and after the treatment for $48 \mathrm{~h}$ with either MLR (light grey columns) or cytokine MIX combination (dark grey columns). The panel shows the expression of the mesenchymal genes snai1, snai2, zeb1 and vimentin (vim), and the epithelial gene E-cadherin (cdh1). Data are shown as mean ( \pm s.d.) and from three independent experiments; ${ }^{\star} P<0.01 ;{ }^{\star \star} P<0.03 ;{ }^{\star \star \star} P<0.05$. Middle panel: Evaluation of EMT changes by flow cytometry on A549 (A), MCF7 (B) and HepG2 (C) cancer cell lines in the same conditions as described above (CTRL, white columns; MLR, light grey columns; MIX, dark grey columns). Data are expressed as marker/isotype fluorescence ratio $(n=3)$ ( \pm s.d.). Dotted line represents the isotype negative control. Statistical significance compared with control condition: ${ }^{*} P<0.01$; ${ }^{\star \star} P<0.03$; ${ }^{* \star \star} P<0.05$. Right panel: Evaluation of EMT changes by immunofluorescence (representative case) on A549 (A), MCF7 (B) and HepG2 (C) cancer cell lines in the same conditions as described above (CTRL, MLR, MIX). E-cadherin (green), vimentin (red), nuclei (blue). 
MLR- or MIX-priming, were co-cultured with stimulated NK cells (Figures $2 \mathrm{~A}-\mathrm{C})$. At the end of co-culture (day +6$)$, NK cells were analysed by flow cytometry to assess viability (left panels) and proliferation rate (right panels). NK cell-mediated lysis of cancer cells was also assessed, without showing significant changes between basal and EMT conditions (Supplementary Figure S2).

NK cell viability after co-culture with A549 cells at basal conditions (CTRL) was higher $(78.3 \pm 5.5 \%)$ than using NK cells alone $(60.3 \pm 10 \%)$ (Figure $2 \mathrm{~A}$, left panel). However, in co-culture conditions with MLR- or MIX-primed A549, NK cell viability decreased significantly and reached comparable levels to the condition with NK cells only (MLR-primed: $65 \pm 1.2 \%$; MIXprimed $66 \pm 2.0 \%$; Figure $2 \mathrm{~A}$, left panel). Viable $\mathrm{NK}$ cell proliferation was also assessed by flow cytometry (Figure 2A, right panel). The co-culture with A549 cells at basal conditions increased NK cell proliferation ( $163 \pm 17 \%$, as compared with basal proliferation values normalised to $100 \%)$. However, when cocultured with MLR- or MIX-primed A549, NK cells proliferated significantly less than in the co-culture with A549 at basal conditions (MLR-primed: $114.2 \pm 1.5 \%$; MIX-primed: $89 \pm 14 \%$ ).

NK cells in co-culture with MCF7 did not show significant changes in viability in any of the culture conditions (NK only: $60.3 \pm 10 \%$; MCF7 CTRL: $68 \pm 19.6 \%$; MLR-primed: $59.6 \pm 13.0 \%$; MIX-primed: $53 \pm 20.0 \%$; Figure $2 \mathrm{~B}$, left panel). By contrast, the proliferation of viable NK cells in co-culture with MCF7, both at basal conditions (CTRL) and with MLR- or MIX-primed MCF7, was reduced as compared with the condition with NK cells only (MCF7 CTRL: $89 \pm 14 \%$; MLR-primed: $52 \pm 2.8 \%$; MIX-primed:
$46 \pm 5.4 \%$; NK cells only: $100 \%$; Figure $2 \mathrm{~B}$, right panel). NK cell proliferation was also significantly reduced by both MLR- and MIX-priming as compared with the co-culture with MCF7 at basal conditions (Figure 2B, right panel).

NK cell viability did not change significantly when cells were cultured with HepG2 at resting conditions and after MLR priming (NK cells only: $60.3 \pm 10 \%$; HepG2 CTRL: $60 \pm 5.5 \%$, MLR-primed: $48 \pm 7.9 \%)$ but decreased in co-culture with MIX-HepG2 cells (MIX-primed: $42 \pm 4.5 \%$ ) (Figure 2C, left panel). Viable NK cell proliferation, as assessed by flow cytometry, was higher when NK cells were co-cultured with MLR- or MIX-primed HepG2, as compared with the condition with NK cells only (MLR-primed: $146.6 \pm 20.2 \%$; MIX-primed: $146 \pm 21.6 \%$; NK only: $100 \%$; Figure $2 \mathrm{C}$, right panel). No significant differences were found among the three co-culture conditions of NK cells with HepG2 cells (HepG2 CTRL: $113 \pm 14.4 \%$; MLR-primed: $146.6 \pm 20.2 \%$; MIX-primed: $146 \pm 21.6 \%$; Figure $2 \mathrm{C}$, right panel).

Cancer cell effects on B cells following EMT. A549, MCF7 and HepG2 cells, either at basal conditions or after EMT induction with MLR- or MIX-priming, were co-cultured with stimulated B cells. At the end of the co-culture (day +4$)$, B cells were analysed by flow cytometry to assess viability and proliferation rate. In addition, the presence of $\mathrm{B}$ regulatory cells $\left(\mathrm{CD} 56^{+}\right.$ $\mathrm{CD} 24^{\mathrm{hi}} \mathrm{CD} 34^{\text {hi }}$ ) was also assessed (Figures $3 \mathrm{~A}-\mathrm{C}$ ).

The viability of $\mathrm{B}$ cells, either cultured alone or in co-culture with A549 before and after EMT induction with MLR- or MIXpriming, did not change significantly (B cells only: $75.6 \pm 9.9 \%$;
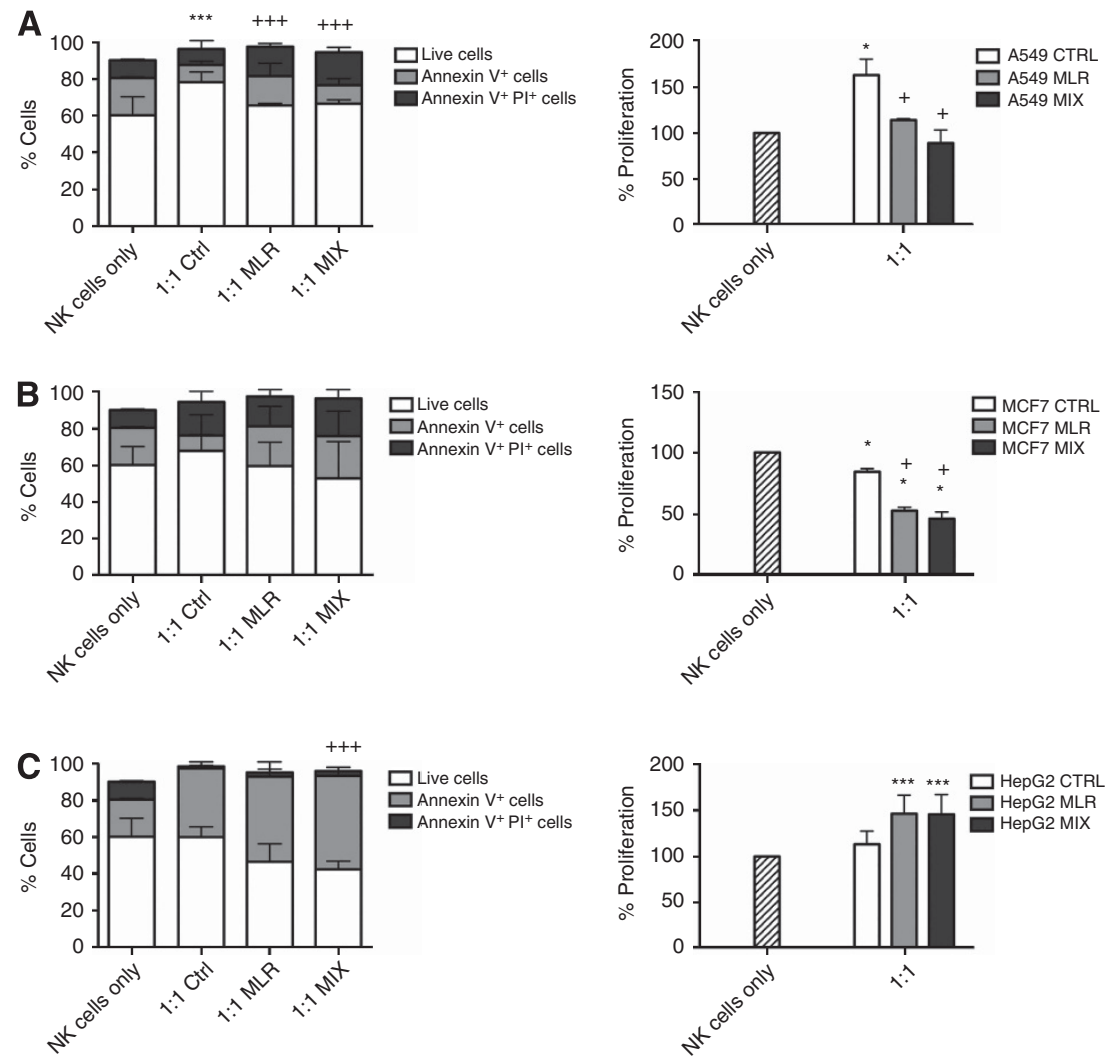

Figure 2. Effect of cancer cells, before and after EMT, on NK cells. Activated NK cells were cultured alone or with (A) A549, (B) MCF7 and (C) HepG2 cells, either at basal conditions (CTRL) or after EMT induction with MLR or MIX priming. The left histograms show NK cell viability; white bar: viable cells; grey bar: annexin $\mathrm{V}^{+}$cells; black bar: annexin $\mathrm{V}^{+} / \mathrm{PI}^{+}$cells. The right histograms show the proliferation rate of viable $\mathrm{NK}$ cells; striped column: NK cells only; white column (CTRL): NK cells co-cultured with cancer cells at basal conditions (control); grey column: NK cells cocultured with MLR-primed cancer cells; black column: NK cells co-cultured with MIX-primed cancer cells. Error bars: s.d. Statistical significance compared with basal condition (NK cell only): ${ }^{*}<0.01$; ${ }^{\star \star} P<0.03$; ${ }^{\star \star \star} P<0.05$. Statistical significance compared with cancer cell CTRL condition: ${ }^{+} P<0.01 ;{ }^{+}+P<0.03 ;{ }^{+}++P<0.05$. 

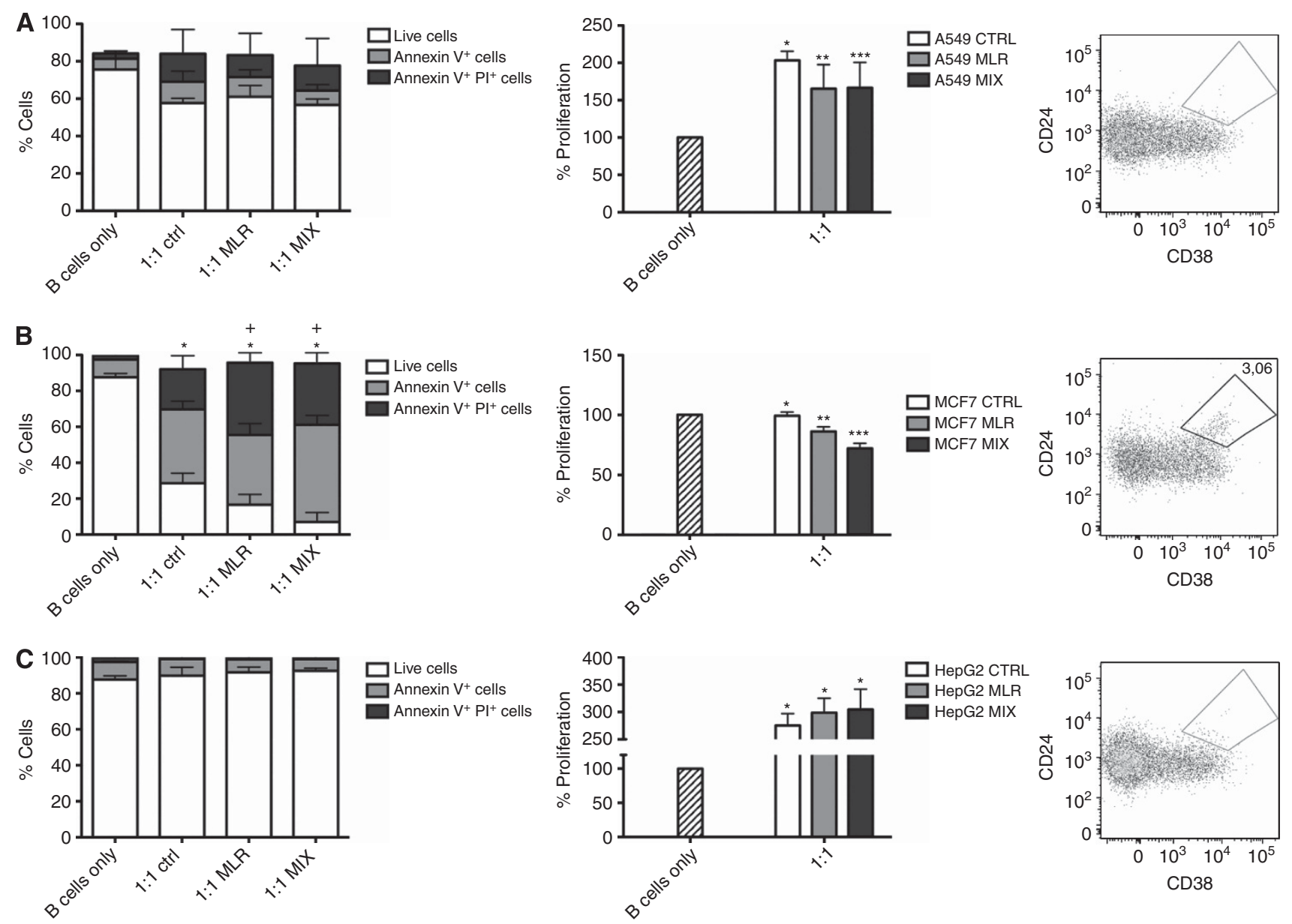

Figure 3. Effect of cancer cells, before and after EMT, on B cells. Activated B cells were cultured alone or with (A) A549, (B) MCF7 and (C) HepG2 cells, either at basal conditions (CTRL) or after EMT induction with MLR or MIX priming. The left histograms show B-cell viability; white bar: viable cells; grey bar: annexin $\mathrm{V}^{+}$cells; black bar: annexin $\mathrm{V}^{+} / \mathrm{PI}^{+}$cells. The middle histograms show the proliferation rate of viable $\mathrm{B}$ cells; striped column: B cells only; white column (CTRL): B cells co-cultured with cancer cells at basal conditions (control); grey column: B cells co-cultured with MLR-primed cancer cells; black column: B cells co-cultured with MIX-primed cancer cells. Dot plots (right panel): gated region of the B-cell regulatory population (representative cases). Error bars: s.d. Statistical significance compared with basal condition (B-cell only): ${ }^{\star} P<0.01$; ${ }^{\star \star} P<0.03 ;{ }^{\star \star \star} P<0.05$. Statistical significance compared with CTRL cancer cell condition: ${ }^{+} P<0.01 ;^{++} P<0.03 ;{ }^{+++} P<0.05$.

A549 CTRL: $57.6 \pm 2.5 \%$; MLR-primed: $61 \pm 6.2 \%$; MIX-primed: $56.6 \pm 3.4 \%$; Figure $3 \mathrm{~A}$, left panel). Viable B-cell proliferation was higher in the presence of cancer cells but not significantly different in the three co-culture conditions (B cells only: 100\%; A549 CTRL: $203 \pm 12.2 \%$; MLR-primed: $165 \pm 32.3 \%$; MIX-primed: $166 \pm 33.9 \%$; Figure $3 \mathrm{~A}$, middle panel). In addition, no $\mathrm{B}$ regulatory cells were found in any of the culture conditions (Figure 3A, right panel).

The viability of $\mathrm{B}$ cells was significantly affected by the coculture with MCF7 cells (B cells only: $87.6 \pm 2.0 \%$; MCF7 CTRL: $28.6 \pm 5.5 \%$; MLR-primed: $16 \pm 5.7 \%$; MIX-primed: $7 \pm 5.3 \%$; Figure 3B, left panel). In addition, the treatment of MCF7 with MLR- and MIX-priming significantly increased the percentage of preapoptotic and dead B cells, as compared with MCF7 in control conditions (Figure 3B, left panel). The effect of MLR- and MIXpriming was also evident by analysing viable B-cell proliferation (B cells only: 100\%; MCF7 CTRL: 99 $\pm 3.2 \%$; MLR-primed: $86.2 \pm 3.8 \%$; MIX-primed: $72.1 \pm 4.3 \%$; Figure $3 \mathrm{~B}$, middle panel). On the contrary, no significant differences were found by comparing the condition with B cells only to B cells co-cultured with MCF7 CTRL. Finally, a significant increase in B regulatory cells was found in the co-culture condition of B cells with MCF7 only after MIX-priming (Figure 3B, right panel).

No significant differences were found in the viability of B cells alone or in co-culture with HepG2 cancer cells in any of the culture conditions (B cells only: $87 \pm 2.0 \%$; HepG2 CTRL: $89.9 \pm 4.6 \%$; MLR-primed: $91.6 \pm 2.89 \%$; MIX-primed: $92.4 \pm 1.56 \%$; Figure 3C, left panel). The analysis of viable B-cell proliferation did not show any significant difference among the different co-culture conditions (CTRL, MLR, MIX) but instead revealed that HepG2 cells, regardless their EMT status, strongly supported B-cell proliferation (B cells only: 100\%; HepG2 CTRL: $275.3 \pm 21.6 \%$; MLR-primed: $298.6 \pm 26.5 \%$; MIX-primed: $304.6 \pm 36 \%$; Figure 3C, middle panel). No B regulatory cells were detected in any of the culture conditions (Figure 3C, right panel).

Cancer cell effects on T cells following EMT. A549, MCF7 and HepG2 cells in control conditions and MLR- or MIX-primed were co-cultured with stimulated T cells (Figures $4 \mathrm{~A}-\mathrm{C}$, respectively). At the end of the co-culture (day +6 ), the immune effector cells were analysed by flow cytometry to measure cell viability (left panel) and proliferation (middle panel). In addition, the presence of $\mathrm{T}$ regulatory cells $\left(\mathrm{CD} 4{ }^{+} \mathrm{CD} 25^{+} \mathrm{FoxP}^{+}\right)$was evaluated (right panel).

The viability of T cells alone or in co-culture with A549 cells did not change significantly among the different conditions ( $\mathrm{T}$ cells only: $74.4 \pm 2.4 \%$; MCF7 CTRL: $44 \pm 15.4 \%$; MLR-primed: $51 \pm 22.2 \%$; MIX-primed: $58 \pm 18.2 \%$; Figure $4 \mathrm{~A}$, left panel). On the contrary, the proliferation of viable $\mathrm{T}$ cells was dramatically 
A

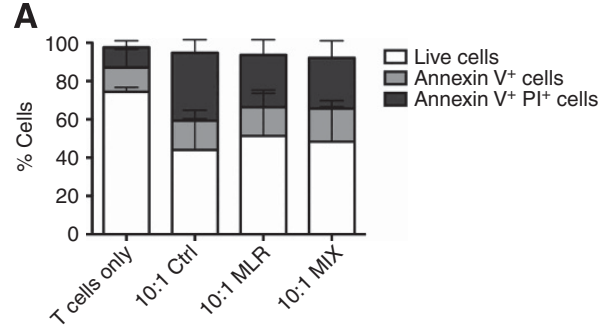

B

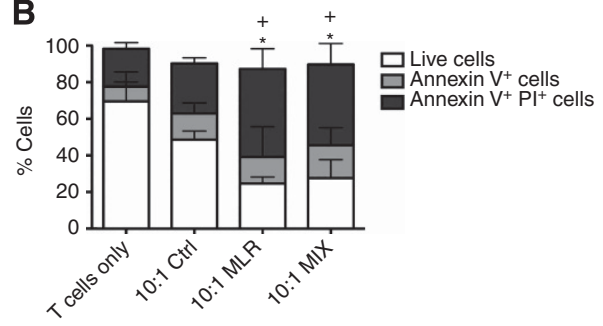

C

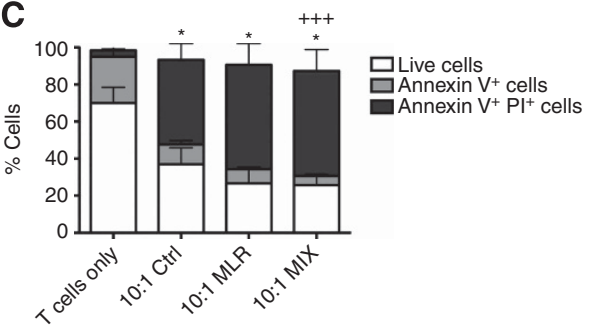

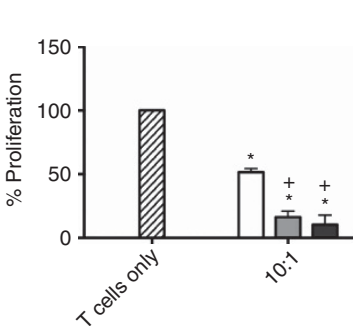
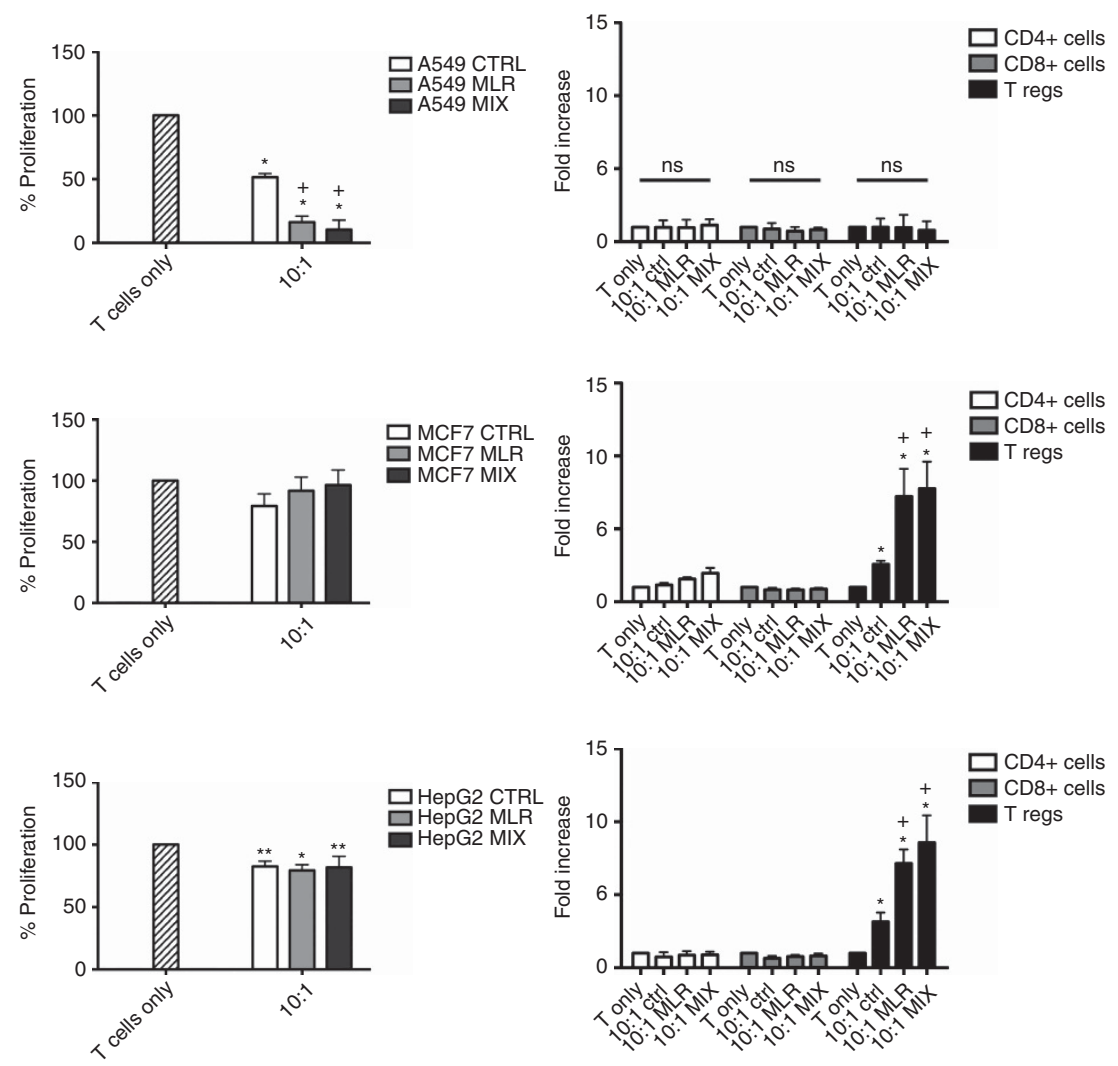

Figure 4. Effect of cancer cells, before and after EMT, on T cells. Activated T cells were cultured alone or with (A) A549, (B) MCF7 and (C) HepG2 cells, either at basal conditions (CTRL) or after EMT induction with MLR or MIX priming. The left histograms show T-cell viability; white bar: viable cells; grey bar: annexin $\mathrm{V}^{+}$cells; black bar: annexin $\mathrm{V}^{+} / \mathrm{PI}^{+}$cells. The middle histograms show the proliferation rate of viable $\mathrm{T}$ cells; striped column: T cells only; white column (CTRL): T cells co-cultured with cancer cells at basal conditions (control); grey column: T cells co-cultured with MLR-primed cancer cells; black column: T cells co-cultured with MIX-primed cancer cells. The right panel shows the composition of T-cell subsets before and after the co-culture with A549 (A), MCF7 (B) and HepG2 (C) following different culture conditions (CTRL, MLR and MIX). White, light grey and black columns: fold increase of $\mathrm{CD} 4^{+} \mathrm{T}$ cells, $\mathrm{CD} 8^{+} \mathrm{T}$ cells, and $\mathrm{T}$ regulatory cells, respectively. Error bars: s.d. Statistical significance compared with basal condition (T-cell only): ${ }^{*} P<0.01 ;{ }^{\star \star} P<0.03$; ${ }^{\star \star \star} P<0.05$. Statistical significance compared with cancer cell CTRL condition: ${ }^{+} P<0.01 ;{ }^{++} P<0.03 ;{ }^{++}+P<0.05$.

affected by co-culture with A549 (T cells only: 100\%; A549 CTRL: $51.5 \pm 2.9 \%$; MLR-primed: $16.1 \pm 4.6 \%$; MIX-primed: $10.4 \pm 7.5 \%$; Figure $4 \mathrm{~A}$, middle panel). The proliferation of viable $\mathrm{T}$ cells was also significantly reduced when T cells were co-cultured with A549 after MLR- and MIX-priming (Figure 4A, middle panel). The analysis of the different T-cell subsets in co-culture with A549 did not reveal any significant change in the balance of $\mathrm{T} \mathrm{CD} 4+$, $\mathrm{CD} 8+$ and $\mathrm{T}$ regulatory cells among the different culture conditions (Figure 4A, right panel).

In the presence of MCF7 cells, T-cell viability did not change significantly ( $\mathrm{T}$ cells only: $69.5 \pm 10 \%$; $\mathrm{T}$ cells in co-culture with MCF7 CTRL: $48.6 \pm 4.5 \%$; Figure 4B, left panel). However, after MLR- and MIX-priming, T-cell viability significantly lowered (T cells only: $69.5 \pm 10 \%$; MCF7 CTRL: $48.6 \pm 4.5 \%$; MLR-primed: $24.7 \pm 3.5 \%$; MIX-primed: $27.5 \pm 10 \%$; Figure $4 \mathrm{~B}$, left panel). Viable T-cell proliferation did not vary in different culture conditions ( $\mathrm{T}$ cells only: 100\%; MCF7 CTRL: 79 $\pm 9.9 \%$; MLR-primed: $91 \pm 11.2 \%$; MIX-primed: $96 \pm 12.3 \%$; Figure $4 \mathrm{~B}$, middle panel). A significant increase in the amount of $\mathrm{T}$ regulatory cells was observed when T cells were co-cultured with MCF7, regardless of their EMT status ( $\mathrm{T}$ cells only: 1\%; MCF7 CTRL: $2.57 \pm 0.25 \%$; MLR-primed: $7.2 \pm 1.8 \%$; MIX-primed: $7.5 \pm 1.8 \%$; Figure $4 \mathrm{~B}$, right panel). However, MLR- and MIX-priming induced a significantly higher amount of $\mathrm{T}$ regulatory cells in comparison to what was observed when T cells were co-cultured with MCF7 in control conditions (Figure 4B, right panel).
Finally, T-cell viability was significantly lower in all the coculture conditions with HepG2 cells (T cells only: $70 \pm 8.4 \%$; HEPG2 CTRL: $37 \pm 8.8 \%$; MLR-primed: $26 \pm 7.6 \%$; MIX-primed: $25 \pm 6.0 \%$; Figure $4 \mathrm{~A}$, left panel). A statistically significant decrease was also observed by comparing the viability of $\mathrm{T}$ cells in coculture with MIX-primed HepG2 vs HepG2 CTRL (Figure 4C, middle panel). Viable T-cell proliferation was reduced regardless of HepG2 EMT status (T cells only: 100\%; HEPG2 CTRL: $82 \pm 4.3 \%$; MLR-primed: $79 \pm 4.6 \%$; MIX-primed: $81 \pm 8.8 \%$; Figure 4C, middle panel). Similarly, a significant increase in the amount of T regulatory cells was observed regardless of HepG2 EMT status (T cells only: $1 \%$; HepG2 CTRL: $3.15 \pm 0.61 \%$; MLR-primed: $7.14 \pm 0.96 \%$; MIX-primed: $8.57 \pm 1.85 \%$; Figure $4 \mathrm{C}$, right panel). However, MLR- and MIX-priming induced a significantly higher amount of T regulatory cells in comparison to what observed when $\mathrm{T}$ cells were co-cultured with HepG2 in control conditions (Figure 4C, right panel).

Mechanisms involved in cancer cell immunomodulation following EMT. As MCF7 and HepG2 had a prevalent pro-apoptotic effect on T cells, while A549 mainly inhibited T-cell proliferation without inducing significant apoptosis, we investigated the involvement of FasL and IDO pathways in the immunomodulatory properties acquired by cancer cells after EMT induction.

FasL protein expression by flow cytometry was undetectable in all the cancer cell lines both before and after EMT induction with 
either MLR- or MIX-priming (data not shown). In addition, ELISA assay did not detect significant concentrations of soluble FasL in the cell supernatants of any of the culture conditions (data not shown).

The involvement of IDO in the immunomodulatory properties of cancer cells was first measured by qRT-PCR (Figure 5A). After MLR- and MIX-priming, all the cell lines significantly upregulated ido1 transcription, particularly following MIX-priming (normalised expression of ido1 in A549 CTRL: 1; in MLR-primed A549: 4313; in MIX-primed A549: 46 666; in MCF7 CTRL: 1; in MLRprimed MCF7: 478; in MIX-primed MCF7: 22 626; in HepG2 CTRL: 1; in MLR-primed HepG2: 400; in MIX-primed HepG2: 4000; Figure 5A). In addition, after EMT induction with MLR- or MIX-priming, ido1 resulted in overexpression at the protein level in all the three cancer cell lines (Figure 5B). In particular, idol level was higher and paralled EMT evidence following MIX-priming, as confirmed by E-cadherin downmodulation and increased expression of Snail1 (Figure 5B). IFN- $\gamma$ was the main inflammatory cytokine responsible for this effect, further enhanced by TNF- $\alpha$. In fact, EMT induction in the absence of IFN- $\gamma$, that is, with either TGF- $\beta$ only or TGF- $\beta+$ TNF- $\alpha$, did not determine a significant IDO-1 upregulation (Supplementary Figure S1), thus paralleling MSC behavior following inflammatory priming (Krampera, 2011).

To test IDO effect, T-cell viability and proliferation assays were repeated with or without the specific inhibitor of IDO, that is, L-1MT. The inhibition of T-cell proliferation with A549 cells in control conditions and after MLR- and MIX-priming was almost completely restored in the presence of L-1MT (A549 CTRL: $51.5 \pm 2.9 \%$; A549 CTRL + L-1MT: $88 \pm 5.5 \%$; MLR-primed A549:
$16.1 \pm 4.6 \%$; MLR-primed A549+L-1MT: 82.6 $\pm 5.8 \%$; MIXprimed A549: 10.4 $\pm 7.5 \%$; MIX-primed A549+L-1MT: $82.3 \pm 7.3 \%$; Figure 5C, left panel). Similar results on T-cell proliferation were obtained by adding L-1MT to the co-culture of T cells with MCF7 and HepG2 cell lines following EMT induction (MLR-primed MCF7: $24.7 \pm 3.5 \%$; MLR-primed MCF7 + L-1MT: $59 \pm 9.43 \%$; MIX-primed MCF7: $27.5 \pm 10 \%$; MIX-primed MCF7 + L-1MT: $62 \pm 9.1 \%$; MLR-primed HepG2: $26 \pm 7.6 \%$; MLR-primed HepG2 + L-1MT: 73.6 \pm 5.2\%; MIX-primed HepG2: $25 \pm 6.0 \%$; MIX-primed HepG2 + L-1MT: $78 \pm 7.9 \%$ ), but not with MCF7 and HepG2 at basal conditions (MCF7 CTRL: $48.6 \pm 4.5 \%$; MCF7 CTRL + L-1MT: $52 \pm 6.9 \%$; HepG2 CTRL: $37 \pm 8.8 \%$; HepG2 CTRL + L-1MT: $45.6 \pm 6.8 \%$; Figure 5C, middle and right panels). As expected, the addition of L-1MT to the culture media did not change significantly the amount of $\mathrm{T}$ regulatory cells induced by the co-culture of T cells with MCF7 and HepG2 (data not shown).

\section{DISCUSSION}

EMT molecular mechanisms have been extensively studied as they are a key step towards tumour development and dissemination through the induction of highly invasive cancer phenotype. In addition, EMT may drive the inflammatory reactions occurring during cancer development and, in turn, is influenced by the inflammatory microenvironment (Mantovani et al, 2008; López-Novoa and Nieto, 2009; Grivennikov et al, 2010). However, how cancer cells may affect the immune system during EMT is still
A

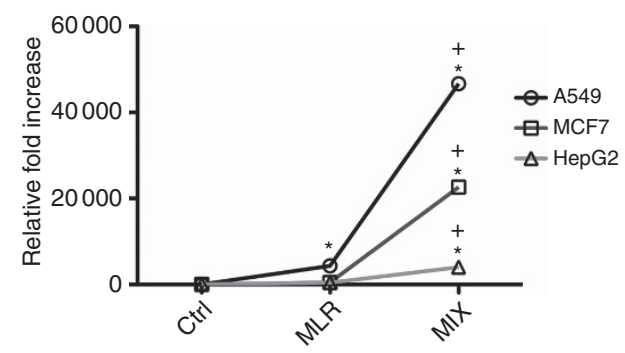

B

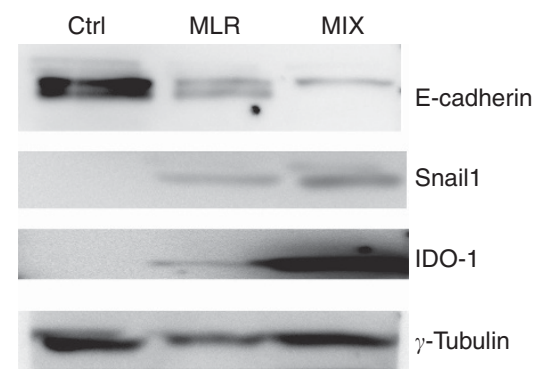

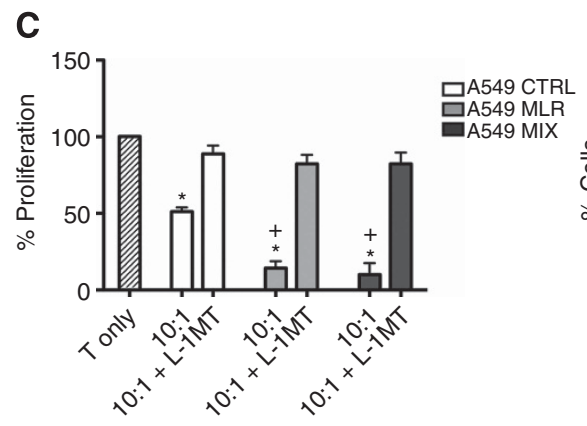
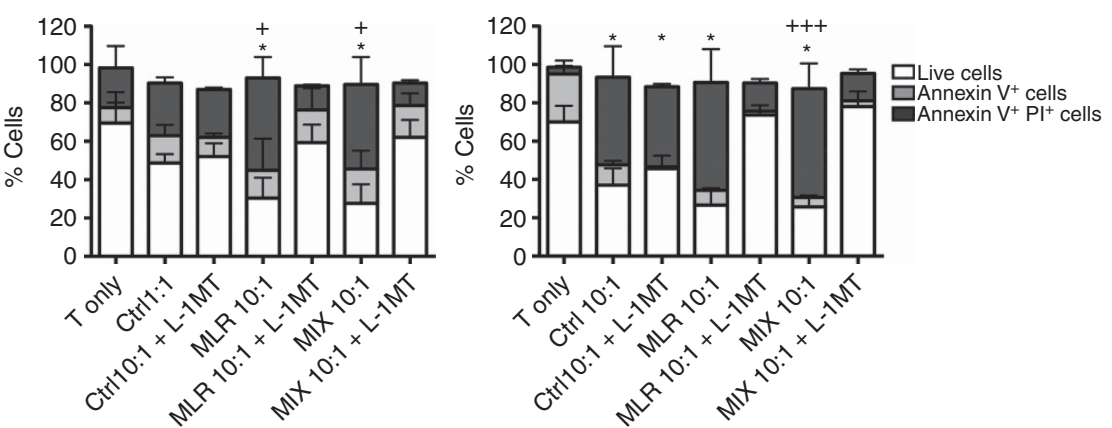

Figure 5. Involvement of IDO. (A) ido1 gene expression in A549 (circle), MCF7 (square) and HepG2 (triangle) cells at basal conditions (CTRL) and after EMT induction with MLR or MIX priming. Graph lines show the relative fold increase expression of ido1. (B) Representative protein expression of E-cadherin, Snail1, Ido1 and $\gamma$-tubulin in A549, MCF7 and HepG2 at basal condition (CTRL) and after EMT induction with MLR or MIX priming. (C) The effect of IDO inhibitor (L-1MT) on T cells, taking into consideration that A549 mainly inhibit T-cell proliferation without inducing significant apoptosis, while MCF7 and HepG2 have a prevalent pro-apoptotic effect on T cells: Left histograms: viable T-cell proliferation rate in presence of A549 cells with or without L-1MT addition (striped column: T cells only; white column: T cells co-cultured with cancer cells at basal conditions; grey column: T cells co-cultured with MLR-primed cancer cells; black column: T co-cultured with MIX-primed cancer cells). Middle histograms: T-cell viability in presence of MCF7 cells with or without L-1MT addition (white bar: viable T cells; grey bar: annexin $\mathrm{V}^{+}$cells; black bar: annexin $\mathrm{V}^{+} / \mathrm{PI}^{+}$ cells); Right histograms: T-cell viability in the presence of HepG2 cells with or without L-1MT addition (white bar: viable T cells; grey bar: annexin $\mathrm{V}^{+}$cells; black bar: annexin $\mathrm{V}^{+} / \mathrm{PI}^{+}$cells). Error bars: s.d. Statistical significance compared with basal condition $\left(\mathrm{T}\right.$-cell only): ${ }^{\star} P<0.01 ;{ }^{\star \star} P<0.03$; ${ }^{\star * \star} P<0.05$. Statistical significance compared with cancer cell CTRL condition: ${ }^{+} P<0.01 ;{ }^{++} P<0.03 ;{ }^{++}+P<0.05$. 
Table 1. Summary of the effects of cancer cell lines after EMT in co-culture with the immune effector cells

\begin{tabular}{|c|c|c|c|c|c|c|c|c|c|}
\hline \multirow[b]{2}{*}{ Cell line } & \multicolumn{2}{|c|}{ NK cells } & \multicolumn{3}{|c|}{ B cells } & \multicolumn{4}{|c|}{ T cells } \\
\hline & Viability & Proliferation & Viability & Proliferation & $B$ regs & Viability & Proliferation & T regs & IDO \\
\hline A549 (lung) & $\downarrow$ & $\downarrow$ & $=$ & $=$ & ND & $=$ & $\downarrow$ & ND & $\uparrow$ \\
\hline MCF7 (breast) & $=$ & $\downarrow$ & $\downarrow$ & $\downarrow$ & $\uparrow$ & $\downarrow$ & $=$ & $\uparrow$ & $\uparrow$ \\
\hline HEPG2 (liver) & $\downarrow$ & $=$ & $=$ & $\uparrow$ & ND & $\downarrow$ & $=$ & $\uparrow$ & $\uparrow$ \\
\hline
\end{tabular}

only partially uncovered (Uyttenhove et al, 2003; Kudo-Saito et al, 2009; Lob et al, 2009a; Joffroy et al, 2010).

In this study, we hypothesised that cancer epithelial cells undergoing EMT following inflammatory priming may acquire at least some of the typical inhibitory properties commonly expressed by MSCs, including IDO activation (Krampera, 2011), which would give some cues to explain their capability of surviving in a potentially hostile inflammatory microenvironment and escaping the specific anti-tumour immune response.

According to our hypothesis, we demonstrate here that EMT can be triggered by cytokines normally produced by the inflammatory microenvironment (TGF- $\beta$, TNF- $\alpha$ and IFN- $\gamma$ ), as well as by the supernatant derived from in vitro immune reactions, such as MLR. Inflammatory-induced EMT promotes a number of variable immune-modulatory mechanisms depending on the cancer cell origin, including: interference with proliferation of $\mathrm{NK}$ and $\mathrm{B}$ cells (A549 and MCF-7 cell lines) and T cells (A549 and HepG2); apoptosis induction on NK cells (A549 and HepG2 cell lines), B cells (MCF7 cell line) and T cells (MCF7 and HepG2 cell lines); and expansion of both regulatory T (MCF7 and HepG2 cell lines) and B cells (MCF7 cell line). All the above-mentioned mechanisms of immune modulation are shared by MSCs of different origin, except apoptosis induction on immune effector cells, which is rarely observed with MSCs (Krampera, 2011; Di Trapani et al, 2013) and therefore seems to be a peculiar feature of cancer cells undergoing EMT (Uyttenhove et al, 2003; Muller et al, 2005; Lob et al, 2009a). In any case, we confirm that IDO pathway after EMT induction by inflammatory stimuli is an important, although not unique, molecular mechanism responsible for T-cell inhibition, as previously shown (Uyttenhove et al, 2003; Muller et al, 2005; Lob et al, 2009a); by contrast, Fas-FasL pathway does not seem to be involved in T-cell apoptosis induction, at least under these experimental conditions.

As previously mentioned, a different pattern of inhibitory mechanisms following inflammation-induced EMT has been found depending on the origin of cancer cell lines. In particular, A549 cells deriving from lung adenocarcinoma appear to influence immune reactions mainly by reducing $\mathrm{NK}$ cell viability and proliferation, and hampering T-cell proliferation, without significant induction of regulatory $\mathrm{B}$ and $\mathrm{T}$ cells. By contrast, MCF-7 cells, deriving from breast cancer, is capable of affecting NK cell proliferation without inducing apoptosis, significantly impair B-cell functions by lowering their viability and proliferation and inducing $\mathrm{B}$ regulatory cells and hamper T-cell response through both pro-apoptotic effects, without modifying proliferation rate, and induction of T regulatory cells. Finally, HepG2 cells, deriving from hepatocarcinoma, clearly hamper T-cell functions by both inhibiting survival and inducing $\mathrm{T}$ regulatory cells; however, they have opposite effects on NK cells, by inducing both cell apoptosis and proliferation, and B cells, whose proliferation is supported, thus probably selecting some NK and B-cell subsets that might be instrumental to HepG2 cell survival (Table 1).

Some of the peculiarities can be explained by the immunophenotypic differences of the three cell lines in the expression, following EMT induction, of class I (upregulated by A549 and MCF7) and II (upregulated by A549 and MCF7) HLA molecules, in the absence of co-stimulatory molecule expression, such as CD80 and CD86 (data not shown), thus leading to the lack of NK cell activation or T- and B-cell anergy (Schwartz, 1990; Diederichsen et al, 2003; Chang et al, 2005). However, other functional properties, such as the induction of B regulatory cells by MCF7 cell line and T regulatory cells by MCF7 and HepG2 cell lines, are probably related to the tissue of origin and the specific molecular pathways triggered by inflammatory-mediated EMT induction. Nevertheless, both B and $\mathrm{T}$ regulatory cells have been recently associated with tumour progression and are reciprocally linked (Olkhanud et al, 2011; Balkwill et al, 2013); in addition, $\mathrm{T}$ regulatory cells may impair survival and proliferation of activated $\mathrm{CD} 4{ }^{+}$and $\mathrm{CD}^{+} \mathrm{T}$ cells (Grossman et al, 2004; Viguer et al, 2004).

Our data further confirm the important role of IDO pathway in T-cell inhibition by cancer cells following inflammation-induced EMT, regardless of their tissue of origin. IDO creates an immunosuppressive microenvironment by depleting tryptophane and accumulating kynurenine metabolites (Fallarino et al, 2006; Platten et al, 2012), and it is a general mechanism of immune modulation shared by MSCs both in normal and cancer microenvironment (Krampera, 2011; Ling et al, 2014). IDO expression has been used in cancer patients as a biomarker to monitor disease activity and response to therapy (Riesenberg et al, 2007; Platten et al, 2012), as its expression is increased in many cancer cell types (Ge et al, 2000a; Tajiri et al, 2003); consequently, specific IDO inhibitors have been suggested for cancer therapy (Lob et al, 2009b).

In summary, our work shows that the acquisition through inflammatory stimuli of mesenchymal phenotype by cancer cells of different origin is associated with the onset of MSC-like immuneregulatory functions and some additional properties, such as apoptosis induction in immune effector cells, which all may lead to tumour immune escape and, consequently, to cancer progression and metastatic dissemination.

\section{REFERENCES}

Balkwill F, Montfort A, Capasso M (2013) B regulatory cells in cancer. Trends Immunol 34(4): 169-173.

Chang CC, Campoli M, Ferrone S (2005) Classical and nonclassical HLA class I antigen and NK cell-activating ligand changes in malignant cells: current challenges and future directions. Adv Cancer Res 93: 189-234.

Di Trapani M, Bassi G, Ricciardi M, Fontana E, Bifari F, Pacelli L, Giacomello L, Pozzobon M, Fèron F, Anversa P, Fumagalli G, Decimo I, Menard C, Tarte K, Krampera M (2013) Comparative study of immune regulatory properties of stem cells derived from different tissues. Stem Cells Dev 22: 2990-3002.

Diederichsen AC, Hjelmborg Jv, Christensen PB, Zeuthen J, Fenger C (2003) Prognostic value of the CD4+/CD8 + ratio of tumour infiltrating lymphocytes in colorectal cancer and HLA-DR expression on tumour cells. Cancer Immunol Immunother 52(7): 423-428.

Fallarino F, Grohmann U, You S, McGrath BC, Cavener DR, Vacca C, Orabona C, Bianchi R, Belladonna ML, Volpi C, Santamaria P, Fioretti MC, Puccetti P (2006) The combined effects of tryptophan starvation and tryptophan catabolites down-regulate $\mathrm{T}$ cell receptor $\zeta$-chain and induce a regulatory phenotype in naive T cells. J Immunol 176: 6752-6761. 
Ge K, Duhadaway J, Sakamuro D, Wechsler-Reya R, Reynolds C, Prendergast GC (2000a) Losses of the tumor suppressor BIN1 in breast carcinoma are frequent and reflect deficits in programmed cell death capacity. Int J Cancer 85: 376-383.

Ge K, Minhas F, DuHadaway J, Mao NC, Wilson D, Buccafusca R, Sakamuro D, Nelson P, Malkowicz SB, Tomaszewski J, Prendergast GC (2000b) Loss of heterozygosity and tumor suppressor activity of Bin1 in prostate carcinoma. Int J Cancer 86: 155-161.

Ge K, DuHadaway J, Du W, Herlyn M, Rodeck U, Prendergast GC (1999) Mechanism for elimination of a tumor suppressor: aberrant splicing of a brain-specific exon causes loss of function of Bin1 in melanoma. Proc Natl Acad Sci USA 96: 9689-9694.

Grivennikov SI, Greten FR, Karin M. Immunity, Inflammation, and Cancer (2010) Cell 140(6): 883-899.

Grossman WJ, Verbsky JW, Barchet W, Colonna M, Atkinson JP, Ley TJ (2004) Human T regulatory cells can use the perforin pathway to cause autologous target cell death. Immunity 4: 589-601.

Joffroy CM, Buck MB, Stope MB, Popp SL, Pfizenmaier K, Knabbe C (2010) Antiestrogens induce transforming growth factor - mediated immunosuppression in breast cancer. Cancer Res 70(4): 1314-1322.

Klymkowsky MW, Savagner P (2009) Epithelial-mesenchymal transition. Am J Pathol 174(5): 1588-1593.

Kotsafti A, Farinati F, Cardin R, Cillo U, Nitti D, Bortolami. M (2012) Autophagy and apoptosis-related genes in chronic liver disease and hepatocellular carcinoma. BMC Gastroenterol 12: 118-129.

Krampera M (2011) Mesenchymal stromal cell 'licensing': a multistep process. Leukemia 25(9): 1408-1414.

Kudo-Saito C, Shirako H, Takeuchi T, Kawakami Y (2009) Cancer metastasis is accelerated through immunosuppression during Snail-induced EMT of cancer cells. Cancer Cell 15(3): 195-206.

Ling W, Zhang J, Yuan Z, Ren G, Zhang L, Chen X, Rabson AB, Roberts AI, Wang Y, Shi Y (2014) Mesenchymal stem cells use IDO to regulate immunity in tumor microenvironment. Cancer Res 74(5): 1576-1587.

Liu J, Hu G, Chen D, Gong A-Y, Soori GS, Dobleman TJ, Chen MX (2013) Suppression of SCARA5 by Snaill is essential for EMT-associated cell migration of A549 cells. Oncogenesis 2(9): 1-10.

Lob S, Konigsrainer A, Zieker D, Brucher BL, Rammensee HG, Opelz G, Terness P (2009a) IDO1 and IDO2 are expressed in human tumors: levo- but not dextro-1-methyl tryptophan inhibits tryptophan catabolism. Cancer Immunol Immunother 58: 153-157.

Lob S, Königsrainer A, Rammensee HG, Opelz G, Terness P (2009b) Inhibitors of indoleamine-2,3-dioxygenase for cancer therapy: can we see the wood for the trees? Nat Rev Cancer 9(6): 445-452.

López-Novoa JM, Nieto MA (2009) Inflammation and EMT: an alliance towards organ fibrosis and cancer progression. EMBO Mol Med 1(6-7): 303-314.

Mantovani A, Allavena P, Sica A, Balkwill F (2008) Cancer-related inflammation. Nature 454: 436-444.
Muller AJ, Du Hadaway JB, Donover PS, Sutanto Ward E, Prendergast GC (2005) Inhibition of indoleamine 2,3-dioxygenase, an immunoregulatory target of the cancer suppression gene Bin1, potentiates cancer chemotherapy. Nat Med 11: 312-319.

Olkhanud PB, Damdinsuren B, Bodogai M, Gress RE, Sen R, Wejksza K, Malchinkhuu E, Westo RP, Biragyn A (2011) Tumor-evoked regulatory b cells promote breast cancer metastasis by converting resting CD $4+\mathrm{T}$ cells to T-regulatory cells. Cancer Res 71: 3505-3515.

Platten M, Wick W, Van den Eynde BJ (2012) Tryptophan catabolism in cancer: beyond IDO and tryptophan depletion. Cancer Res 72(21): 5435-5440.

Riesenberg R, Weiler C, Spring O, Eder M, Buchner A, Popp T, Castro M, Kammerer R, Takikawa O, Hatz RA, Stief CG, Hofstetter A, Zimmermann W (2007) Expression of indoleamine 2,3-dioxygenase in tumor endothelial cells correlates with long-term survival of patients with renal cell carcinoma. Clin Cancer Res 13: 6993-7002.

Schwartz RH (1990) A cell culture model for T lymphocyte clonal anergy. Science 248: 1349.

Tajiri T, Liu X, Thompson PM, Tanaka S, Suita S, Zhao H, Maris JM, Prendergast GC, Hogaty MD (2003) Expression of a MYCN-interacting isoform of the tumor suppressor BIN1 is reduced in neuroblastomas with unfavorable biological features. Clin Cancer Res 9: 3345-3355.

Thiery JP, Acloque H, Huang RYJ, Nieto MA (2009) Epithelial-mesenchymal transitions in development and disease. Cell 139(5): 871-890.

Uyttenhove C, Pilotte L, Theate I, Stroobant V, Colau D, Parmentier N, Boot T, Van den Eynde BJ (2003) Evidence for a tumoral immune resistance mechanism based on tryptophan degradation by indoleamine 2,3-dioxygenase. Nat Med 9: 1269-1274.

Vetter G, Saumet A, Moes M, Vallar L, Le Béchec A, Laurini C, Sabbah M, Arar K, Theillet C, Lecellier CH, Friederich E (2010) miR-661 expression in SNAI1-induced epithelial to mesenchymal transition contributes to breast cancer cell invasion by targeting Nectin-1 and StarD10 messengers. Oncogene 29: 4436-4448.

Viguer M, Lemaitre F, Verola O, Cho MS, Gorochov G, Dubertret L, Bachelez H, Kourisky P, Ferradini L (2004) Foxp3 expressing $\mathrm{CD} 4+\mathrm{CD} 25$ high regulatory $\mathrm{T}$ cells are overrepresented in human metastatic melanoma lymph nodes and inhibit the function of Infiltrating T cells. J Immunol 173(2): 1444-1453.

Voulgari A, Pintzas A (2009) Epithelial-mesenchymal transition in cancer metastasis: Mechanisms, markers and strategies to overcome drug resistance in the clinic. BBA - Reviews on Cancer 1796(2): 75-90.

This work is published under the standard license to publish agreement. After 12 months the work will become freely available and the license terms will switch to a Creative Commons AttributionNonCommercial-Share Alike 4.0 Unported License.

Supplementary Information accompanies this paper on British Journal of Cancer website (http://www.nature.com/bjc) 